\title{
Effect of linker distribution in the photocatalytic activity of multivariate mesoporous crystals
}

Belén Lerma-Berlanga,† Carolina R. Ganivet,† Neyvis Almora-Barrios,†.Yong Peng,§ Josep Albero,§ Oscar Fabelo,॥ Javier GonzálezPlatas, ${ }^{\perp}$ Hermenegildo García, ${ }^{\S}$ Natalia M. Padial,,${ }^{\ominus}$, and Carlos Martí-Gastaldo ${ }^{\dagger}$

† Functional Inorganic Materials Team, Instituto de Ciencia Molecular (ICMol), Universitat de València, Paterna 46980, València, Spain.

§ Instituto Universitario de Tecnología Química CSIC-UPV, Universitat Politècnica de València, Av. De Los Naranjos s/n, València, Spain

$\perp$ Departamento de Física. Instituto Universitario de Estudios Avanzados en Física Atómica, Molecular y Fotónica (IUDEA). MALTA Consolider Team. Universidad de La Laguna, Avda. Astrofísico Fco. Sánchez s/n, La Laguna, Tenerife, E-38204, Spain.

॥ Institut Laue Langevin, 71 avenue des Martyrs, CS 20156, Grenoble, Cedex 9 38042, France.

ABSTRACT: The use of Metal-Organic Frameworks as crystalline matrices for the synthesis of multiple component or multivariate solids by the combination of different linkers into a single material has emerged as a versatile route to tailor the properties of single component phases or even access new functions. This approach is particularly relevant for $\mathrm{Zr}_{6}$ - $\mathrm{MOFs}_{\mathrm{O}}$ due to the synthetic flexibility of this inorganic node. However, the majority of materials is isolated as polycrystalline solids, which are not ideal to decipher the spatial arrangement of parent and exchanged linkers for the formation of homogeneous structures or heterogeneous domains across the solid. Here we use High-Throughput methodologies to optimize the synthesis of single crystals of UiO-68 and UiO-68-TZDC, a photoactive analogue based on a tetrazine dicarboxylic derivative. The analysis of the single linker phases reveals the necessity of combining both linkers to produce multivariate frameworks that combine efficient light sensitization, chemical stability and porosity, all relevant to photocatalysis. We use solvent assisted linker exchange reactions to produce a family of UiO-68-TZDC\% binary frameworks, which respect the integrity and morphology of the original crystals. Our results suggest that the concentration of TZDC in solution controls the distribution of this linker in the sibling crystals for a random mixture or the formation of core-shell domains. We also demonstrate how the possibility of generating an asymmetric distribution of both linkers has a negligible effect on the electronic structure and optical bandgap of the solids but controls their performance for drastic changes in the photocatalytic activity towards proton or methyl viologen reduction.

\section{Introduction}

The discovery of the family of $\mathrm{Zr}(\mathrm{IV}) \mathrm{UiO}$-type Metal-Organic Frameworks (MOFs) by Lillerud and co-workers in 2008 introduced a versatile platform for the design of MOFs with excellent stability. ${ }^{1}$ Since then, the use of monocarboxylic acids as modulators ${ }^{2}$ combined with the flexibility of $\left[\mathrm{Zr}_{6} \mathrm{O}_{4}(\mathrm{OH})_{4}\left(\mathrm{CO}_{2}\right)_{12}\right]$ clusters acting as inorganic nodes, has enabled to gain chemical control over the design of isoreticular frameworks with linear dicarboxylic linkers for a huge family of porous materials with tailorable micro/mesoporosity and application in catalysis, gas storage or separation technologies. ${ }^{3,4}$ Unfortunately, most of these materials can be only synthesised as polycrystalline solids highlighting the difficulties to isolate single crystals. This problem is even more severe for the mesoporous derivatives, that require the use of elongated linkers with very poor solubility in the organic solvents used in the synthesis. This is possibly why the synthesis of single crystals of UiO-68 by using $\mathrm{H}_{2}$ TPDC ([1,1':4',1"'-terphenyl]-4,4"'-dicarboxylic acid) linkers remains still elusive, and all crystals available rely on amine, alcohol or alkyl derivatives with subsequent changes to the porosity or physical properties of the pristine material.5,6,7
The availability of single crystals is particularly important in understanding the dynamic exchange reactions often used to produce multivariate frameworks by post-synthetic (PSE) ${ }^{8}$ or solvent-assisted linker exchange (SALE) ${ }^{9}$. The porosity of the framework and the concentration of the exchanged linker control the diffusion of the last. As result, these variables can

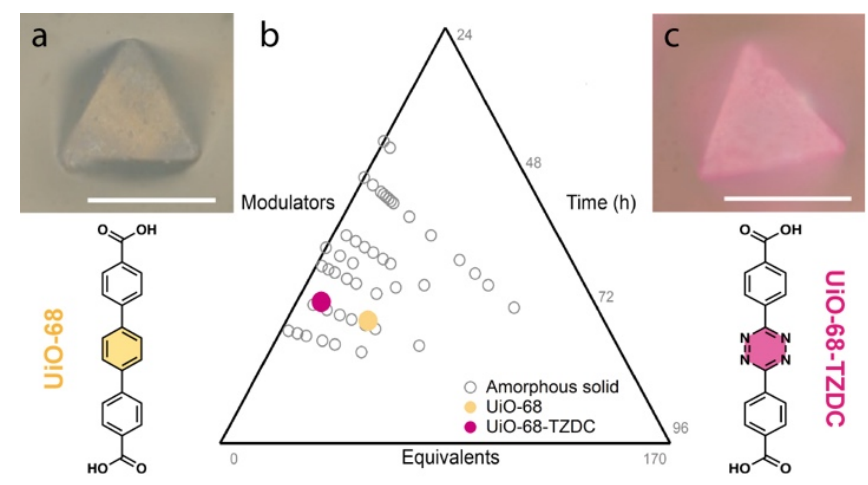

Figure 1. a) Size and morphology of UiO-68. Scale bars correspond to $50 \mu \mathrm{m}$. b) Summary of the synthetic conditions explored with automated HT synthesis to identify crystal 
forming conditions. c) Size and morphology of photoactive UiO68-TZDC crystals. Scale bars correspond to $25 \mu \mathrm{m}$.

impose changes to the microstructure and distribution of exchanged: parent linkers in the framework. Both features might modify the physical properties and performance of the MOF in specific applications. This phenomenon was originally demonstrated for microporous MOF-5, UMCM-8 and UiO-66,10 but there are no precedents for mesoporous frameworks. Built upon or recent experience for the challenging chemistry of titanium ${ }^{11-13}$, here we use High-Throughput (HT) synthetic methodologies to optimize the conditions required for isolating single crystals of pristine UiO-68 and its photoactive analogue UiO-68-TZDC, based on 4,4'-(1,2,4,5-tetrazine-3,6diyl)dibenzoic acid ( $\mathrm{H}_{2}$ TZDC). The availability of crystals is used to analyse the effect of TZDC concentration in the formation of binary frameworks by SALE. Our results confirm that the crystals evolve from an ordered core-shell microstructure of TZDC:TPDC linkers into a random distribution for increasing equivalents of the exchanged linker. By correlating the electronic structure of UiO-68-TZDC $\mathrm{C}_{\mathrm{x}}$ solids with their photocatalytic activity, we demonstrate the impact of linker distribution over the photocatalytic performance of multivariate mesoporous crystals.

We designed a first experiment for optimizing the formation of UiO-68 crystals that accounted for systematic variations of temperature $\left(90,100\right.$ or $120^{\circ} \mathrm{C}$ ), reaction time ( 48 or 72 hours) and equivalents of acid modulator: trifluoroacetic acid (TFA), acetic acid (HAc), benzoic acid (BA) or formic acid (FA). The metal:linker stoichiometry, zirconium source and solvent were fixed according to the protocols adopted by the community to $1: 1, \mathrm{ZrCl}_{4}$ and $N, N$-dimethylformamide (DMF) ${ }^{12}$ for a library of 100 reactions. We used a FLEX SHAKE HT workstation from Chemspeed(C) for robotic dispensing of solids and liquids combined with a 96-well powder x-Ray diffraction (PXRD) plate. This helped maintaining a high reproducibility and accelerating the systematic screening of reaction parameters based on the crystallinity and PXRD fingerprints of the products. See Supplementary section S2 for more experimental details. As shown in the ternary diagram in Figure 1b, crystals of UiO-68 were only formed by reaction with 40 equivalents of TFA at $100^{\circ} \mathrm{C}$ during $72 \mathrm{~h}$. The solid was isolated as pale-yellow crystals with octahedral morphology and sizes ranging from 20 to $50 \mu \mathrm{m}$ (Figure 1a). To challenge the versatility of these synthetic conditions we next synthesised $\mathrm{H}_{2}$ TZDC by replacing the central phenyl ring in $\mathrm{H}_{2}$ TPDC with a tetrazine version. Tetrazine derivates are electroactive molecules capable of undergoing reversible transformation into a stable anion-radicals. As result, their fluorescence can be electrochemically switched for applications in light generation, sensors and polymers. ${ }^{14,15}$ These features can be also translated to MOFs for generating photoactive frameworks by reticulation of tetrazine derivatives. ${ }^{16,17}$ Despite the poorer solubility of the tetrazine linker, the conditions optimized for TPDC only required a slight reduction of the modulator equivalents, from 40 to 10 , to isolate UiO-68 TZDC as pink octahedral crystals with sizes ranging from 10 to $20 \mu \mathrm{m}$ (Figure 1c).

UiO-68 and UiO-68-TZDC crystallize in the cubic space group Fm-3m ( $a=32.71$ and $32.47 \AA$ respectively). They display an expanded cubic closed-packed structure for the characteristic 12-connected $f c u$ topology of the UiO family. ${ }^{1}$ See

a
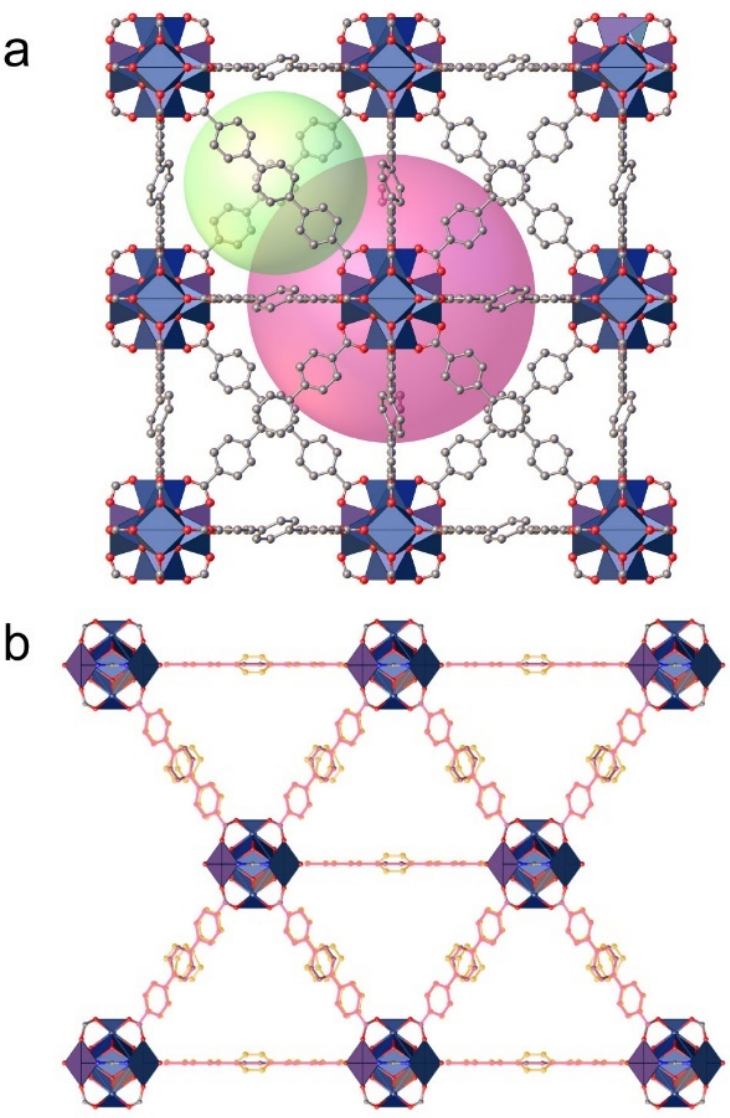

Figure 2. a) Structure of UiO-68 along the c axis showing the accesible volume corresponding to octahedral (purple sphere) and tetrahedral voids (green). b) Overlay of the two crystallographic-distinct linker structures in (011) showing the slight differences that result from the replacement of TPDC with TZDC.

Supplementary section $\mathbf{S 3}$ for more details. The impact of the linker replacement in the porosity metrics is almost negligible for octahedral and tetrahedral voids of 27.3 and $19.3 \AA$ (Figure $\mathbf{2 a , b}$ ). The main structural differences associated to the incorporation of TZDC are reflected on changes to the coordination bonds. The $\mathrm{Zr}-\mu_{3} \mathrm{O}$ and $\mathrm{Zr}-\mu_{2} \mathrm{O}$ distances in the metal-oxo cluster and the carboxylate group are elongated up to $0.012 \AA$ in UiO-68-TZDC due to the electron withdrawing nature of the tetrazine core (Table S2). As for the internal structure of the linkers, the carboxylate groups remain coplanar in both cases but the central ring presents a certain degree of disorder. We collected crystallographic data at room temperature (UiO-68-TZDC) and $100 \mathrm{~K}$ (UiO-68). The disorder is present in both cases which suggests this is intrinsic to the crystallographic packing of the solids and not thermal in origin. The effect of the tetrazine core is more notable in the thermal and chemical stability of the frameworks. Compared to UiO-68 that decomposes in air above $500{ }^{\circ} \mathrm{C}$, the thermogravimetric analysis of UiO-68-TZDC shows a gradual decomposition from 250 to $450{ }^{\circ} \mathrm{C}$ indicative of the oxidation of the tetrazine core at 
high temperatures (Figure S13). The hydrolytical stability of the solids was evaluated by incubation in water for 24 hours. The PXRDs after this treatment suggest that the structure of
UiO-68 remains intact whereas UiO-68-TZDC undergoes partial amorphization.

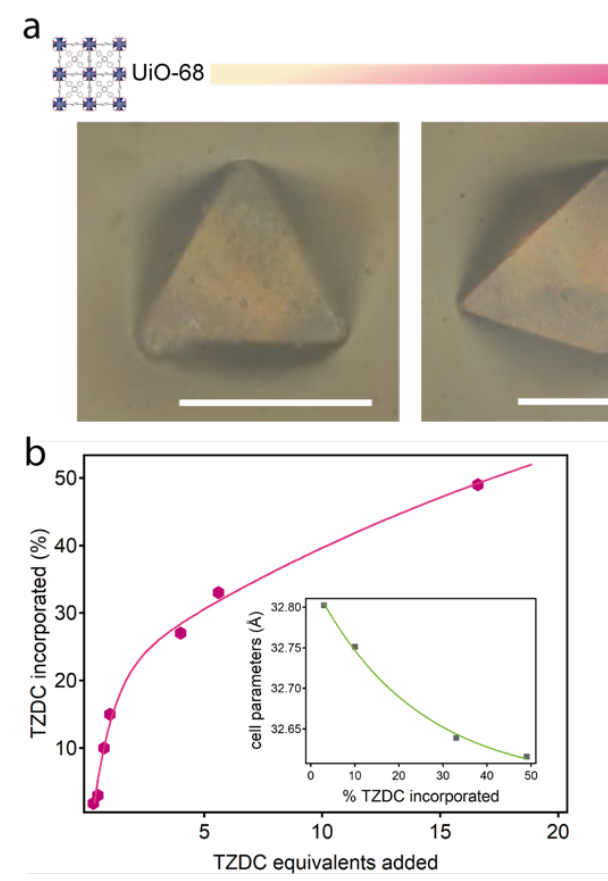

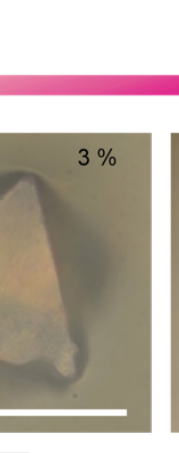

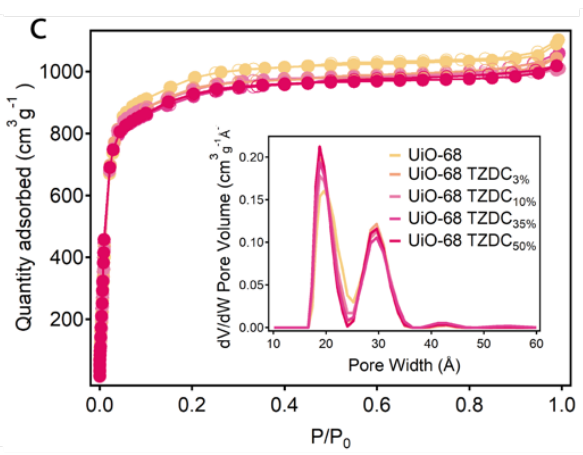

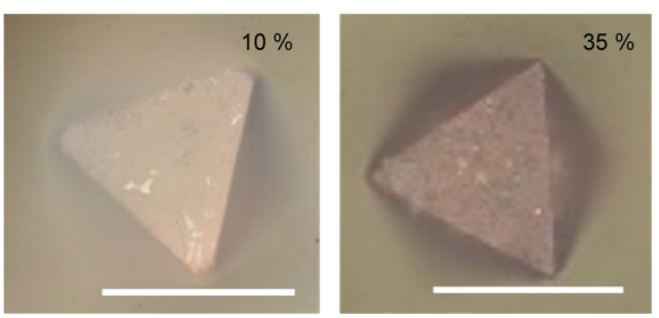

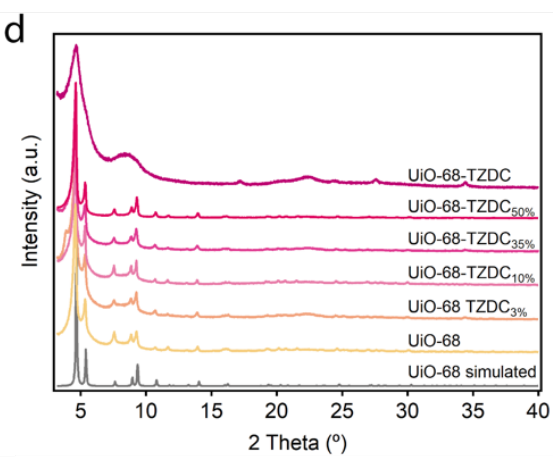

Figure 3. a) Optical microscope image of UiO-68 TZDC\% samples. Scale bars correspond to $50 \mu \mathrm{m}$. b) TZDC equivalents used in the synthesis (left axis). Reduction cell parameters as result of incorporation TZDC. c) N2 sorption isotherms at $77 \mathrm{~K}$ and PSD for UiO-68 TZDC\% samples. (Filled marker were used for adsorption and empty marker for desorption). d) PXRD of the solids as after soaked $\mathrm{H}_{2} \mathrm{O} /$ methanol (4:1) for 24 hours.

(Figure S14). This agrees well with the weakening of the elongated coordination bonds in the last for feebler metal joints more prone to hydrolysis.

We next analysed the effect of the linker in tuning the structural robustness of the solids for optimizing the process of activation, a critical step to access the expected surface areas. ${ }^{18}$ We selected a range of solvents to examine the effect of changes in coordinating ability, polarity or surface tension in the collapse of porosity (Table S4, Figure S15). ${ }^{19}$ Methanol exchange leads to amorphization in both cases likely due to its ability to react with $\mathrm{Zr}_{6}$ nodes. ${ }^{20}$ In turn, acetone and DMF are respectful with the structure of UiO-68 but provoke the collapse of UiO-68-TZDC. This suggests a negative effect of the electron-deficient heterocycle in maximizing the interaction with polar solvents for a framework more susceptible to the capillaryforces generated in the activation process. To confirm this point, we used hexane for minimizing the interaction with the framework due to its low surface tension $\left(17.9 \mathrm{mN} \cdot \mathrm{m}^{-1}\right.$ at $25^{\circ} \mathrm{C}$ ) and small dipole moment (0.08 D). Exchange with hexane for 5 days, followed by drying in anhydrous conditions, for avoiding humidity and reducing capillary forces $^{21}$ is respectful with the structure of both materials. See Supplementary section \$4.6 for more details. Both samples were degassed overnight at $60^{\circ} \mathrm{C}$ and $10^{-6}$ Torr before gas analysis. As shown in Figure S17, they display a non-hysteretic, type-I $\mathrm{N}_{2}$ adsorption isotherms with multi- point BET surface areas in excellent agreement with the expected values, 4028 and $4289 \mathrm{~m}^{2} \cdot \mathrm{g}^{-1}$ for UiO-68 and UiO68-TZDC respectively. ${ }^{1}$

The pore size distributions (PSDs) calculated by using NonLinear Density Functional Theory (NLDFT) models reveal a narrow distribution of two types of mesopores centered at 2.0 and $3.0 \mathrm{~nm}$, consistent with the pore size values calculated from the crystallographic data available (Table S5). Additional measurements collected for samples exchanged with wet hexane or methanol show a significant drop in surface area of 30 and $80 \%$ for UiO-68-TZDC, confirming the importance of minimizing solvent-framework interaction to access the expected porosity (Figure S17). Note that this is only critical for the activation step. After that, the solid can be exposed to open air with no impact over its surface area. $\mathrm{CO}_{2}$ adsorption measurements reveal a modest uptake at $273 \mathrm{~K}$ of around 50 $\mathrm{cm}^{3} \cdot \mathrm{g}^{-1}$. The calculated isosteric heats of adsorption confirm the positive effect of the nitrogenated core in UiO-68-TZDC for stronger interaction with the guest (Qst $=31 \mathrm{~kJ} \cdot \mathrm{mol}^{-1}$ ) (Figure S18). The properties of the single linker phases reveal the necessity of combining both units in the same material to produce a photoactive material without compromising its chemical stability or porosity, both important to photocatalytic applications. We used a HT library of reactions to produce multivariate MOFs 22 by direct combination of TPDC and TZDC with systematic control of ratio, temperature, concentration and mixture of solvents, All attempts produced amorphous solids with negligible incorporation of TZDC, suggesting 
important differences in the reactivity of the linkers with Zr(IV) (Supplementary section S5.1). Based on their structural similarity, we decided to use post-synthetic linker exchange methods instead. By using UiO-68 crystals as precursors, we prepared a family of UiO-68-TZDC\% multivariate solids by SALE of varying equivalents of the tetrazine linker in $\mathrm{DMF}$ at $80^{\circ} \mathrm{C}$ for 3 hours. We used a base $\left(\mathrm{Et}_{3} \mathrm{~N}\right)$ to deprotonate $\mathrm{H}_{2} \mathrm{TZDC}$ and favour the linker exchange (Supplementary section S5.2). ${ }^{23}$ UiO-68-TZDC $\%$ crystals were digested and analysed by ${ }^{1} \mathrm{H}$ NMR (Figure S21). The integration of the resonances for aromatic protons confirm the presence of both linkers and reveal a fine control over the exchange rate from 3 to $50 \%$, concomitant with the colour of the crystals (Figure 3a). The FT-IR spectra of the samples shows a shift of $25 \mathrm{~cm}^{-1}$ in the $v_{\mathrm{C}=0}$ vibration compared to the free linker. These rules out the trapping of TZDC in the mesopores of the MOF and confirms that the NMR quantification is representative of the actual incorporation of the linker to the framework (Figure S24). We used these techniques to analyse the maximum exchange ratio possible. Beyond UiO-68 $\mathrm{TZDC}_{50 \%}$, reactions with more than 20 equivalents of TZDC show clear evidences of ineffective linker exchange in the final products (Figure S31). As shown in Figure 3b, UiO-68-TZDC $3-50 \%$ crystals show the same logarithmic rate of TZDC incorporation with the number of equivalents observed for other Zr-MOFs. ${ }^{24}$ Phase purity was confirmed by PXRD Le Bail refinement of all solids. We observe a compression of the unit cell parameters that correlates well with the TZDC \% incorporated. All solids display permanent porosity with negligible changes to their surface area and PSD compared to the single linker phases (Figure 3c). SEM analysis rules out any morphological damage during linker exchange. All crystals retain the octahedral morphology and size of the
UiO-68 seed, suggesting that the incorporation of TZDC operates in the crystal and does not involve their dissolution/recrystallization (Figure S28).

Besides respecting the structure and porosity of the pristine solids, the coexistence of TPDC and TZDC in multivariate UiO68 crystals allows for reaching a fine balance between chemical stability and photoactivity. We soaked Ui0-68-TZDC $3-50 \%$ crystals in $\mathrm{H}_{2} \mathrm{O}: \mathrm{CH}_{3} \mathrm{OH}$ mixtures $(4: 1, v / v \%)$ for 24 hours. These methanolic solutions are often used to test the photocatalytic activity of MOFs in hydrogen evolution reactions.11,12,13, Compared to Ui0-68-TZDC that becomes amorphous, all solids retain their original structure with negligible changes in their PXRD patterns regardless the TZDC\% incorporated (Figure 3d). Linker sensitization is an efficient way to maximize the photocatalytic activity of MOFs with visible by taking advantage of their synthetic/structural tunability. This strategy is dominated by porphyrins, 25 functionalized organic linkers 26,27 or the incorporation of ruthenium or iridium complexes. ${ }^{28} s$-Tetrazine linkers are also excellent candidates in this regard due to the combination of one-electron reversible reduction behaviour and visible-light sensitization. To understand the effect of tetrazine doping, we calculated the electronic structure of the hybrid frameworks by using density functional theory (DFT, Supplementary section S6.1). Figure 4a shows a comparison of the electronic density of states diagram of pristine UiO-68 and UiO-68-TZDC $4 \%$, that corresponds to the incorporation of one TZDC linker per unit cell. In UiO-68, the position the highest occupied crystalline orbitals (HOCO) and the lowest unoccupied crystalline orbital (LUCO) is dominated by the aromatic $2 p$ orbitals of TPDC. The introduction of a single TZDC linker per cell yields a LUCO that a

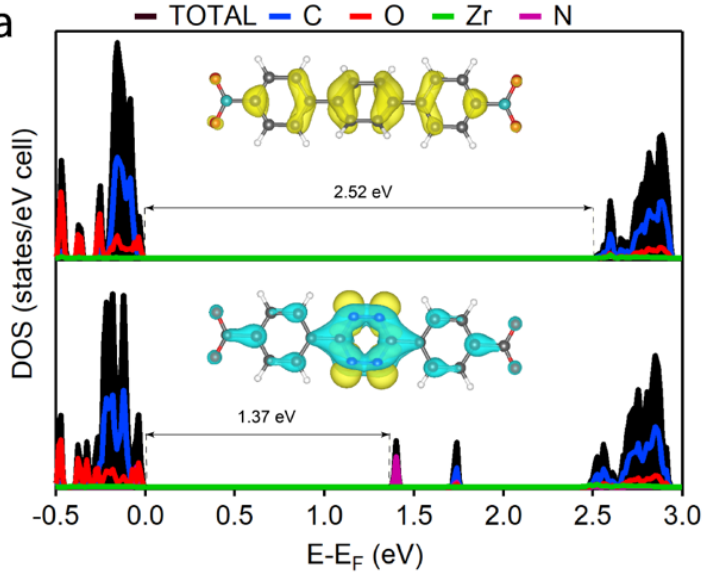

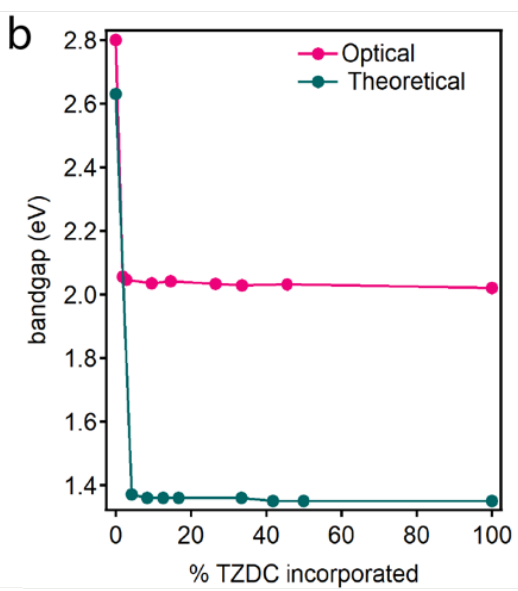

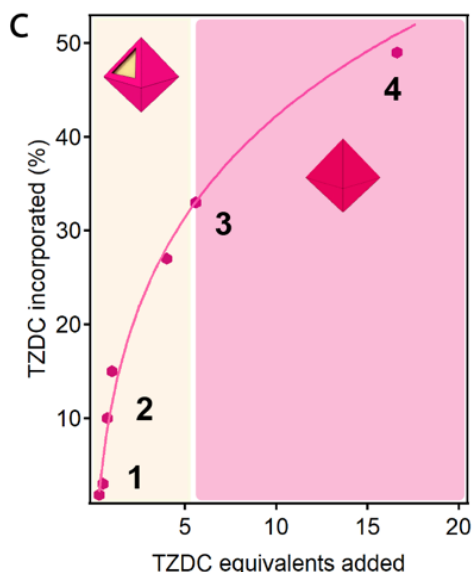

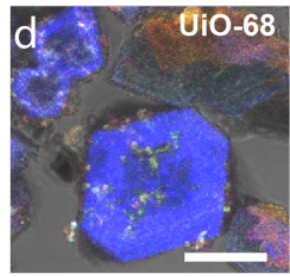
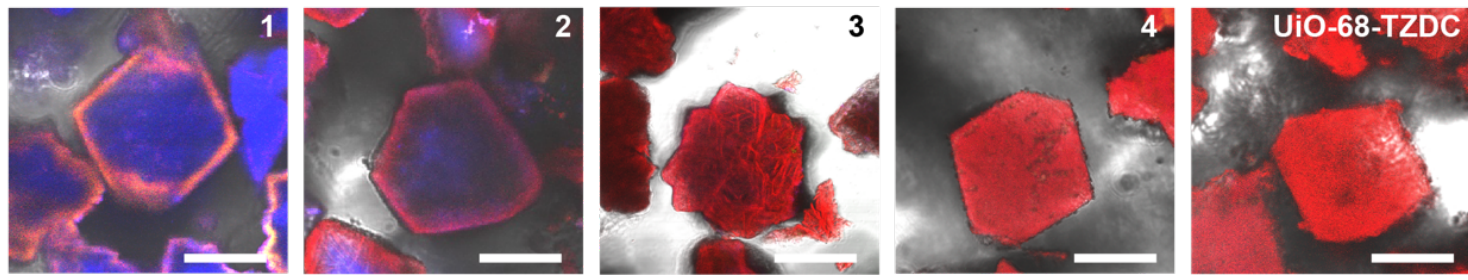

Figure 4. a) Total (DOS, black) and projected (PDOS for each atoms) density of states for pristine UiO-68 (top) and UiO-68-TZDC4\%, (bottom) calculated by using PBE functional, this is a qualitative approach; the electronic structures could not be computed at the HSE06 level because of the system size. Inset: orbitals contributing to the HOCO and LUCO localised on ligand. b) Match between the band gap values' trend described by experimental results and computer calculations. c) Spatial linker arrangement in Ui0-68-TZDC\% samples (deep orange area for core-shell distribution and pink area for random distribution). d) Fluorescence confocal microscope's 
images of polish UiO-68 samples reveal the resultant microstructures. Scale bars correspond to 25 and $10 \mu \mathrm{m}$ for UiO-68 counterparts and UiO-68-TZDC respectively.

is also dominated by the linker but shows clear differences. The contribution of the $2 \mathrm{p} \mathrm{N}$ and $\mathrm{C}$ orbitals from the tetrazine ring in UiO-68-TZDC $4 \%$ reduces the band gap to $1.37 \mathrm{eV}$ compared to the $2.52 \mathrm{eV}$ in UiO-68. We carried out additional calculations by systematically increasing the TZDC content up to 50\% (12 linkers per unit cell). As shown in Figure 4a, the position of the LUCO is insensitive to increasing levels of doping and a single linker replacement is sufficient to dominate the electronic structure and optical properties of the hybrids. This effect is very similar to the modification of the band gap with monoaminated terephtalic linkers reported for MIL-125, that also saturates for the replacement of one linker per unit cell.26 To confirm the value of our computational simulations we used UV-Vis solid reflectance measurements to estimate the optical band gap of UiO-68-TZDC $3-50 \%$ by using the Kubelka Munk function. We observe an excellent match between the trend described by experimental results and computed band gap values, that further confirm the effectiveness of TZDC sensitization at very small doping levels (Figure 4b). (Supplementary section S6.2). To further analyse the spatial arrangement of parent and exchanged linkers in the crystal we carried out fluorescence studies with a confocal microscope. Images were taken at different heights to account for the threedimensional distribution of active TZDC and inactive TPDC linkers when excited with a green filter $(\lambda=559 \mathrm{~nm})$. Comparison of UiO-68-TZDC at 3 and 35\% doping levels suggests a higher concentration of TZCD linkers at the surface of the first based on more intense fluorescence at the edges and vertices of the crystal (Supplementary section S7 and movie S1-S2). For a clearer view of this phenomenon we used an ultramicrotome to dissect and polish the section of the crystals. This experiment confirms that the crystals show an asymmetric distribution of linkers for the formation of coreshell/TPDC-TZDC architectures between 3-20\% whereas this distribution becomes random from 35\% (Figure 4c-d). This confirms the ability of UiO-type materials to produce chemically diverse arrangements of functionalities after PSE as demonstrated with Raman and fluorescence imaging for UiO66 and 67, respectively. ${ }^{10,29}$ Our results points out to a chemically diverse set of crystals with comparable optical band gaps ideal to investigate the effect of linker distribution over their photocatalytic activity. To the best of our knowledge, this remains still an open question for multivariate MOFs. We hypothesised that light absorption might be more favourable for asymmetric core-shell crystals compared to their random distribution even for lower doping levels. The higher concentration of photoactive linkers in the surface of the crystal for the first case might reduce scattering for more favourable light absorption. To investigate this possibility, we evaluated the photocatalytic performance of UiO-68-TZDC ${ }_{10 \%}$ and UiO-68-TZDC $35 \%$ for the reduction of methyl viologen (MV) and the hydrogen evolution reaction (HER). As shown in Figure 5a, the asymmetric distribution of photoactive TZDC and inactive TPDC linkers promotes a much more efficient reduction of $\mathrm{MV}$ for $\mathrm{UiO}-68-\mathrm{TZDC}_{10 \%}$ despite the reduced concentration of TZDC. This same trend is observed for the HER in a methanolic mixture(Figure 5b). Compared to the negligible activity of UiO-68-TZDC $35 \%$, UiO-68-TZDC $10 \%$ shows a linear increase in the $\mathrm{H}_{2}$ production up to a maximum of ca.
$40 \mu \mathrm{mol} \cdot \mathrm{g}^{-1}$ after 6.5 hours. It is worth commenting that related MOF-based photocatalysts, such as UiO-66, have shown negligible $\mathrm{H}_{2}$ production in the absence of Pt nanoparticles or other co-catalysts, in good agreement with the production observed for Ui0-68-TZDC $35 \%{ }^{30-32}$ This boost in performance is concomitant to an increase in the quantum efficiency of close to 15 times higher as result of the formation of core-shell domains.
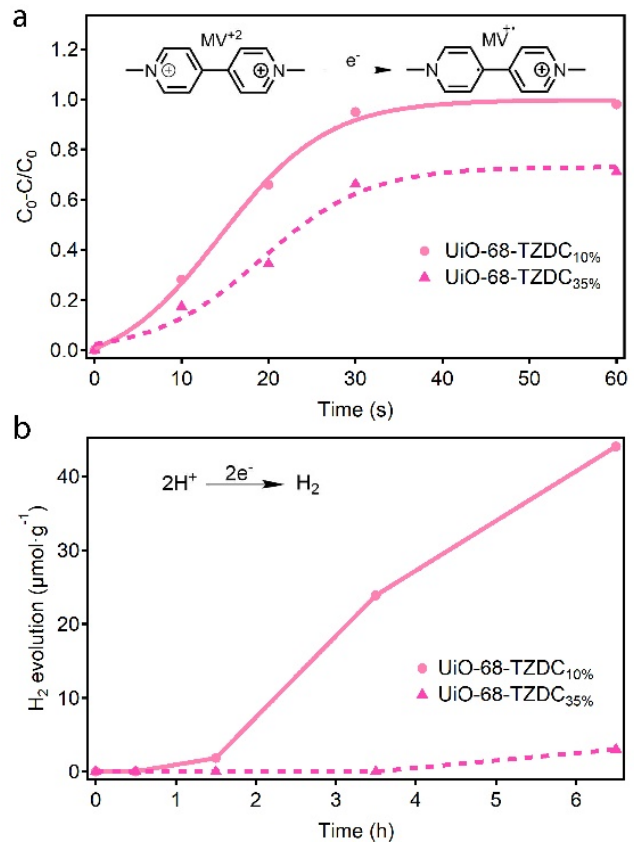

Figure 5. a) Photocatalytic $\mathrm{H}_{2}$ production after 6.5 hours of irradiation for UiO-68-TZDC $10 \%$ (pink circles) and UiO-68TZDC $_{35 \%}$ (deep pink triangles). b) Photocatalytic methyl viologen reduction yields in 1 hour of reaction for UiO-68TZDC $_{10 \%}$ and UiO-68-TZDC $35 \% .1 \mathrm{mg} / \mathrm{mL}$ for a) and $0.2 \mathrm{mg} / \mathrm{mL}$ for b) of catalyst, light source UV-vis xenon lamp $\mathrm{H}_{2} \mathrm{O} /$ methanol (4:1).

Compared to the synthetic design of multiple component MOFs, which often relies only on the nature and relative percentage of the linkers combined as synthetic variables to tune the properties of the resulting materials, our results demonstrate also the impact of linker distribution as an efficient tool to code their function. We are currently exploring this possibility to maximize the sensitization of multivariate titanium frameworks. Compared to Zr-MOFs for which photostimulated processes are mainly restricted to the linker, $33 \mathrm{Ti}$ is more prone to induce ligand to metal charge transfer for higher photocatalytic efficiencies. ${ }^{13,27}$

\section{ASSOCIATED CONTENT}

\section{Supporting Information}

The Supporting Information is available free of charge on the ACS Publications website. 
Synthetic and experimental details; physical characterization and supporting tables and figures (PDF)

X-ray crystallographic data for UiO-68 and UiO-68-TZDC (CIF) Movies S1 and S2: Confocal images of UiO-68 TZDC3\% and 35\% showing the evolution of the fluorescence across the crystal.

\section{AUTHOR INFORMATION}

\section{Corresponding Authors}

*carlos.marti@uv.es

*nmpadial@ugr.es

ORCID

Belén Lerma-Berlanga: 0000-0003-3686-8576

Carolina R. Ganivet: 0000-0002-4738-6460

Neyvis Almora-Barrios: 0000-0001-5269-2705

Josep Albero: 0000-0002-4841-7206

Oscar Fabelo: 0000-0001-6452-8830

Javier Gonzales Platas: 0000-0003-3339-2998

Hermenegildo García: 0000-0002-9664-493X

Natalia M. Padial: 0000-0001-6067-3360

Carlos Martí-Gastaldo: 0000-0003-3203-0047

\section{Present Address}

- Departamento de Química Inorgánica, Universidad de Granada, Avenida Fuentenueva S/N, 18071 Granada, Spain

\section{Notes}

The authors declare no competing financial interest. CCDC contains the supplementary crystallographic data for this paper.

\section{ACKNOWLEDGMENT}

This work was supported by the EU (ERC Stg Chem-fs-MOF 714122) and Spanish government (CTQ2017-83486-P, RTI2018-098568-A-I00 \& CEX2019-000919-M). B.L.-B. thanks the Spanish government for a FPU (FPU16/04162). N.M.P. thanks the European Union for a Marie Skłodowska-Curie Global Fellowship (H2020-MSCA-IF-2016-GF-749359EnanSET). JGP thanks to the SIDIX at Servicios Generales de Apoyo a la Investigación (SEGAI) at La Laguna University.

\section{REFERENCES}

(1) Cavka, J. H.; Jakobsen, S.; Olsbye, U.; Guillou, N.; Lamberti, C.; Bordiga, S.; Lillerud, K. P. A New Zirconium Inorganic Building Brick Forming Metal Organic Frameworks with Exceptional Stability. J. Am. Chem. Soc. 2008, 130, 13850-13851.

(2) Schaate, A.; Roy, P.; Godt, A.; Lippke, J.; Waltz, F.; Wiebcke, M.; Behrens, P. Modulated Synthesis of ZrBased Metal-Organic Frameworks: From Nano to Single Crystals. Chem. - Eur. J. 2011, 17, 6643-6651.

(3) Furukawa, H.; Cordova, K. E.; O’Keeffe, M.; Yaghi, O. M. The Chemistry and Applications of Metal-Organic Frameworks. Science. 2013, 341,1230444.
Yuan, S.; Feng, L.; Wang, K.; Pang, J.; Bosch, M.; Lollar, C.; Sun, Y.; Qin, J.; Yang, X.; Zhang, P.; et al. Stable MetalOrganic Frameworks: Design, Synthesis, and Applications. Adv. Mater. 2018, 30, 1-35.

Li, W. Y.; Yang, S.; Li, Y. A.; Li, Q. Y.; Guan, Q.; Dong, Y. Bin. Synthesis of an MOF-Based $\mathrm{Hg}^{2+}$-Fluorescent Probe: Via Stepwise Post-Synthetic Modification in a Single-Crystal-to-Single-Crystal Fashion and Its Application in Bioimaging. Dalt. Trans. 2019, 48, 16502-16508.

Tan, C.; Han, X.; Li, Z.; Liu, Y.; Cui, Y. Controlled Exchange of Achiral Linkers with Chiral Linkers in $\mathrm{Zr}$ Based UiO-68 Metal-Organic Framework. J. Am. Chem. Soc. 2018, 140, 16229-16236.

Gui, B.; Hu, G.; Zhou, T.; Wang, C. Pore Surface Engineering in a Zirconium Metal-Organic Framework via Thiol-Ene Reaction. J. Solid State Chem. 2015, 223, 79-83.

Kim, M.; Cahill, J. F.; Fei, H.; Prather, K. A.; Cohen, S. M. Postsynthetic Ligand and Cation Exchange in Robust Metal-Organic Frameworks. J. Am. Chem. Soc. 2012, $134,18082-18088$.

Karagiaridi, O.; Bury, W.; Sarjeant, A. A.; Stern, C. L.; Farha, O. K.; Hupp, J. T. Synthesis and Characterization of Isostructural Cadmium Zeolitic Imidazolate Frameworks via Solvent-Assisted Linker Exchange. Chem. Sci. 2012, 3, 3256-3260.

(10) Boissonnault, J. A.; Wong-Foy, A. G.; Matzger, A. J. CoreShell Structures Arise Naturally during Ligand Exchange in Metal-Organic Frameworks. J. Am. Chem. Soc. 2017, 139, 14841-14844.

(11) Castells-Gil, J.; Padial, N. M.; Almora-Barrios, N.; Albero, J.; Ruiz-Salvador, A. R.; González-Platas, J.; García, H.; Martí-Gastaldo, C. Chemical Engineering of Photoactivity in Heterometallic Titanium-Organic Frameworks by Metal Doping. Angew. Chem. Int. Ed. 2018, 130, 8589-8593.

Castells-Gil, J.; M. Padial, N.; Almora-Barrios, N.; Da Silva, I.; Mateo, D.; Albero, J.; García, H.; Martí-Gastaldo, C. De Novo Synthesis of Mesoporous Photoactive Titanium(IV)-Organic Frameworks with MIL-100 Topology. Chem. Sci. 2019, 10, 4313-4321.

(13) Padial, N. M.; Castells-Gil, J.; Almora-Barrios, N.; Romero-Angel, M.; Da Silva, I.; Barawi, M.; GarcíaSánchez, A.; De La Peña O’Shea, V. A.; Martí-Gastaldo, C. Hydroxamate Titanium-Organic Frameworks and the Effect of Siderophore-Type Linkers over Their Photocatalytic Activity. J. Am. Chem. Soc. 2019, 141, 13124-13133.

Clavier, G.; Audebert, P. S -Tetrazines as Building Blocks for New Functional Molecules and Molecular Materials. Chem. Rev. 2010, 110, 3299-3314. 
Jang, H. S.; Jana, S.; Blizzard, R. J.; Meeuwsen, J. C.; Mehl, R. A. Access to Faster Eukaryotic Cell Labeling with Encoded Tetrazine Amino Acids. J. Am. Chem. Soc. 2020, 142, 7245-7249

(16) Feng, L.; Lo, S.; Wang, S.; Lu, K.; Zhou, H.; Feng, L.; Lo, S.; Tan, K.; Li, B.; Yuan, S.; et al. An EncapsulationRearrangement Strategy to Integrate Superhydrophobicity into Mesoporous Metal-Organic Frameworks An Encapsulation-Rearrangement Strategy to Integrate Superhydrophobicity into Mesoporous Metal-Organic Frameworks. Matter 2020, 2, 988-999.

(17) Vinu, M.; Sivasankar, K.; Prabu, S.; Han, J. L.; Lin, C. H.; Yang, C. C.; Demel, J. Tetrazine-Based Metal-Organic Frameworks as Scaffolds for Post-Synthetic Modification by the Click Reaction. Eur. J. Inorg. Chem. 2019, 7, 461-466.

(18) Dodson, R. A.; Wong-Foy, A. G.; Matzger, A. J. The Metal-Organic Framework Collapse Continuum: Insights from Two-Dimensional Powder X-Ray Diffraction. Chem. Mater. 2018, 30, 6559-6565

(19) Ayoub, G.; Islamoglu, T.; Goswami, S.; Friščić, T.; Farha, O. K. Torsion Angle Effect on the Activation of UiO Metal-Organic Frameworks. ACS Appl. Mater. Interfaces 2019, 11, 15788-15794.

(20) Yang, D.; Bernales, V.; Islamoglu, T.; Farha, O. K.; Hupp, J. T.; Cramer, C. J.; Gagliardi, L.; Gates, B. C. Tuning the Surface Chemistry of Metal Organic Framework Nodes: Proton Topology of the Metal-Oxide-Like $\mathrm{Zr}_{6}$ Nodes of UiO-66 and NU-1000. J. Am. Chem. Soc. 2016, 138, 15189-15196.

(21) Mondloch, J. E.; Katz, M. J.; Planas, N.; Semrouni, D.; Gagliardi, L.; Hupp, J. T.; Farha, O. K. Are $\mathrm{Zr}_{6}$-Based MOFs Water Stable? Linker Hydrolysis vs. CapillaryForce-Driven Channel Collapse. Chem. Commun. 2014, 50, 8944-8946.

(22) Deng, H.; Doonan, C. J.; Furukawa, H.; Ferreira, R. B.; Towne, J.; Knobler, C. B.; Wang, B.; Yaghi, O. M. Multiple functional groups of varying ratios in metal-organic frameworks. Science. 2010, 327, 846-850.

(23) Nickerl, G.; Senkovska, I.; Kaskel, S. Tetrazine Functionalized Zirconium MOF as an Optical Sensor for Oxidizing Gases. Chem. Commun. 2015, 51, 2280-2282.

(24) Marreiros, J.; Caratelli, C.; Hajek, J.; Krajnc, A.; Fleury, G.; Bueken, B.; De Vos, D. E.; Mali, G.; Roeffaers, M. B. J.; Van Speybroeck, V.; et al. Active Role of Methanol in Post-Synthetic Linker Exchange in the Metal-Organic Framework UiO-66. Chem. Mater. 2019, 31, 13591369.
Khimyak, Y. Z.; Wiper, P. V.; Darwent, J. R.; Rosseinsky, M. J. A Water-Stable Porphyrin-Based Metal-Organic Framework Active for Visible-Light Photocatalysis. Angew. Chemie - Int. Ed. 2012, 51, 7440-7444.

Hendon, C. H.; Tiana, D.; Fontecave, M.; Sanchez, C.; D’Arras, L.; Sassoye, C.; Rozes, L.; Mellot-Draznieks, C.; Walsh, A. Engineering the Optical Response of the Titanium-MIL-125 Metal-Organic Framework through Ligand Functionalization. J. Am. Chem. Soc. 2013, 135, 10942-10945.

Chambers, M. B.; Wang, X.; Ellezam, L.; Ersen, O.; Fontecave, M.; Sanchez, C.; Rozes, L.; Mellot-Draznieks, C. Maximizing the Photocatalytic Activity of MetalOrganic Frameworks with Aminated-Functionalized Linkers: Substoichiometric Effects in MIL-125- $\mathrm{NH}_{2}$. J. Am. Chem. Soc. 2017, 139, 8222-8228.

(28) Elcheikh Mahmoud, M.; Audi, H.; Assoud, A.; Ghaddar, T. H.; Hmadeh, M. Metal-Organic Framework Photocatalyst Incorporating Bis(4'-(4Carboxyphenyl)-Terpyridine)Ruthenium(II) for Visible-Light-Driven Carbon Dioxide Reduction. J. Am. Chem. Soc. 2019, 141, 7115-7121.

(29) Schrimpf, W.; Jiang, J.; Ji, Z.; Hirschle, P.; Lamb, D. C.; Yaghi, O. M.; Wuttke, S. Chemical Diversity in a MetalOrganic Framework Revealed by Fluorescence Lifetime Imaging. Nat. Commun. 2018, 9, 1647.

(30) Silva, C. G.; Luz, I.; Llabrés I Xamena, F. X.; Corma, A.; García, H. Water Stable Zr-Benzenedicarboxylate Metal-Organic Frameworks as Photocatalysts for Hydrogen Generation. Chem. - Eur. J. 2010, 16, 1113311138.

(31) Lin, R.; Shen, L.; Ren, Z.; Wu, W.; Tan, Y.; Fu, H.; Zhang, J.; $\mathrm{Wu}, \quad$ L. Enhanced Photocatalytic Hydrogen Production Activity via Dual Modification of MOF and Reduced Graphene Oxide on CdS. Chem. Commun. 2014, 50, 8533-8535.

(32) Wang, R.; Gu, L.; Zhou, J.; Liu, X.; Teng, F.; Li, C.; Shen, Y.; Yuan, Y. Quasi-Polymeric Metal-Organic Framework UiO-66/g- $\mathrm{C}_{3} \mathrm{~N}_{4}$ Heterojunctions for Enhanced Photocatalytic Hydrogen Evolution under Visible Light Irradiation. Adv. Mater. Interfaces 2015, 2, 1500037.

(33) Nasalevich, M. A.; Hendon, C. H.; Santaclara, J. G.; Svane, K.; Van Der Linden, B.; Veber, S. L.; Fedin, M. V.; Houtepen, A. J.; Van Der Veen, M. A.; Kapteijn, F.; et al. Electronic Origins of Photocatalytic Activity in d0 Metal Organic Frameworks. Sci. Rep. 2016, 6, 23676 


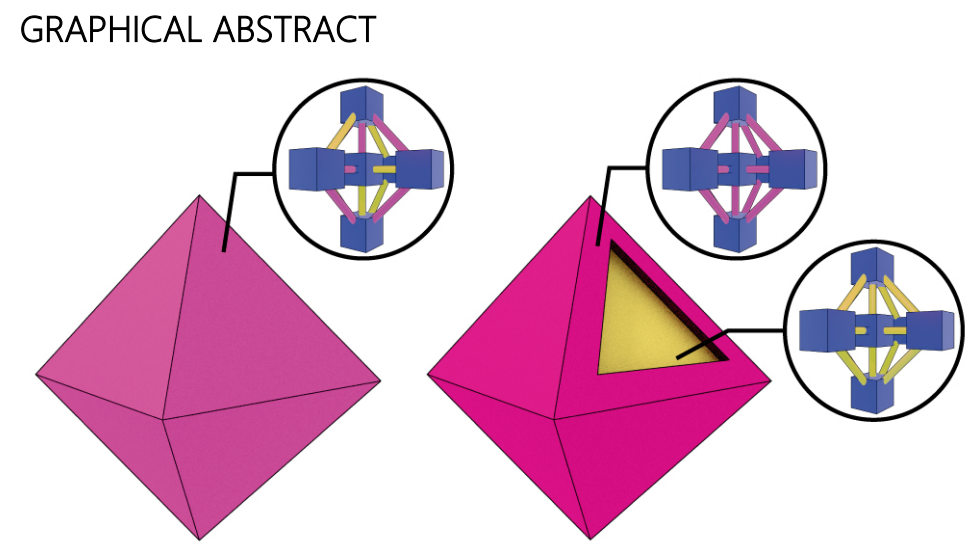




\section{Disclaimer}

This document is the unedited Author's version of a Submitted Work that was subsequently accepted for publication in Journal of American Chemical Society, copyright (c) American Chemical Society after peer review.

To access the final edited and published work see: https://pubs.acs.org/doi/abs/10.1021/jacs.0c09015 